\title{
Magnetic ionic liquid aqueous two-phase system coupled with high performance liquid chromatography: A rapid approach for determination of chloramphenicol in water environment
}

\author{
Tian Yao, Shun Yao*
}

\section{ABSTRACT}

A novel organic magnetic ionic liquid based on guanidinium cation was synthesized and characterized. A new method of magnetic ionic liquid aqueous twophase system (MILATPs) coupled with high-performance liquid chromatography (HPLC) was established to preconcentrate and determine trace amount of chloramphenicol (CAP) in water environment for the first time. In the absence of volatile organic solvents, MILATPs not only has the excellent properties of rapid extraction, but also exhibits a response to an external magnetic field which can be applied to assist phase separation. The phase behavior of MILATPs was investigated and phase equilibrium data were correlated by Merchuk equation. Various influencing factors on CAP recovery were systematically investigated and optimized. Under the optimal conditions, the preconcentration factor was 147.2 with the precision values $(\mathrm{RSD} \%)$ of $2.42 \%$ and $4.45 \%$ for intra-day $(n=6)$ and inter-day $(n=6)$, respectively. The limit of detection (LOD) and limit of quantitation (LOQ) were $0.14 \mathrm{ng} \mathrm{mL}-1$ and $0.42 \mathrm{ng} \mathrm{mL}-1$, respectively. Fine linear range of $12.25 \mathrm{ng} \mathrm{mL}^{-1} \sim 2200 \mathrm{ng} \mathrm{mL}^{-1}$ was obtained. Finally, the validated method was successfully applied for the analysis of CAP in some environmental waters with the recoveries for the spiked samples in the acceptable range of $94.6 \% \sim 99.72 \%$. Hopefully, MILATPs is showing great potential 
to promote new development in the field of extraction, separation and pretreatment of various biochemical samples.

Keywords: magnetic ionic liquid; aqueous two-phase; chloramphenicol; preconcentration; microextraction

\section{Introduction}

Pretreatment of various analytes is the most challenging and time-consuming step in an analytical procedure [1]. Currently, there are many available extraction procedures. For example, conventional solid-phase extraction (SPE) [2] and liquidliquid extraction (LLE) [3] have been used for pre-concentration and clean-up in the 
analysis process as efficient techniques to separate and concentrate various substrates. Besides the advantages shown in their successful applications, they also have some limitations, such as high cost, time-consuming operation, use of large amount of organic solvents which generally are toxic and hazardous to organisms and the environment [4]. As a result, new methods for sample preparation continue to be developed [5]. Among them, dispersive liquid-liquid microextraction (DLLME) was introduced by Assadi et al. as a simple extraction and rapid pre-concentration method [6]. This technique has been widely applied to analyze trace amount of various compounds in environment, food and drink samples for its good repeatability, less extraction time, high recovery as well as high enrichment factor [7-10]. However, volatile organic solvent was also used as disperser solvent which is not environmentally friendly when large scale of samples is pretreated. Shi et al. applied magnetic nanoparticles in the DLLME system to simplify the extraction and separation of target analytes by employing an external magnetic field [11]. Nevertheless, additional desorption procedure was required; it increased time and operational difficulty. Addition of desorption solvent might probably reduce the enrichment factor, and recovery could not be maintained consistently at the high level during the reuse of sorbents.

Aqueous two-phase system (ATPs) is a specific thermodynamic mixture which is usually formed by two different compositions mixed with water. As a promising alternative for traditional organic-water solvent extraction system, it was first proposed in the 20th century [12]. Compared with the above mentioned methods, ATPs is more environmentally friendly without any use of harmful volatile organic solvent in the whole process, and it has been widely used to separate and purify 
proteins [13], antibiotics [14-15], metal ions [15] and dyes [16]. In recent years, some ATPs containing ionic liquids have been being developed and proved to have promising extraction capacity in many separation applications [17-19]. Rogers and his co-workers demonstrated the ability of ILs to form ATPSs in the presence of inorganic salts for the first time in 2003 [20]. IL-salt aqueous two-phase systems (ILATPs) have shown many advantages, such as low viscosity, little emulsion, rapid phase separation and environmentally compatibility [21]. It has been successfully applied as a green, non-toxic and sensitive pretreatment technique for various samples [22]. However, ILATPs still revealed a shortage of low isolation efficiency of two phases because of small density difference [23].

Recently, applications of magnetic room temperature ionic liquid (MRTIL) become a new field and a hotspot of research in sample extraction and concentration techniques [24-27]. Some ILs were immobilized on the surface of magnetic nanoparticles to form functional solid materials and used in magnetic solid-phase extraction [28-29]. These systems not only employ environmentally friendly IL as extractant, but also use magnetic separation technology to simplify separation. However, organic solvent is still introduced in desorption procedure and desorption is really extra tedious operation. Lately, a novel class of genuine magnetic ionic liquids with single-component was discovered. The first example is 1-butyl-3methylimidazolium tetrachloroferrate $\left(\left[\mathrm{C}_{4} \mathrm{mim}\right]\left[\mathrm{FeCl}_{4}\right]\right)$, which was firstly reported in 2004 [30]. These magnetic task-specific ILs exhibit a strong response to a magnetic field [31] and have already been successfully applied in many fields especially in fuel desulfurization and separation processes [32-34]. Recently, a few literatures have reported about the application of MILs on the pretreatment and concentration of target 
analytes in single drop microextraction (SDME) [33] and dispersive liquid-liquid microextraction (DLLME) systems [35-36]. The introduction of MILs can enhance pretreatment efficiency by means of an external magnetic field. However, magnetic SDME is still time-consuming; the use of organic solvent in magnetic DLLME is not that eco-friendly. All the above MILs of great application prospect contain metal elements such as Fe, Co, Dy et al. Another kind of MIL is non-metal organic MIL based on a radical ion, and the first one was synthesized and proposed by Yoshida and coworkers in 2007 [37]. Afterwards, bimagnetic ionic liquid with an organic magnetic center was prepared and efficiently applied in catalysis [38]. Up to now there exist so few organic magnetic ionic liquids. More applications of them were never found in the literatures up to now.

Chloramphenicol (CAP) is an effective antibiotic against a wide range of Grampositive and Gram-negative bacteria in both human and animals [39]. Because of its serious toxicity in humans, CAP is completely banned in food-producing animals within the EU [40]. CAP is easily released or excreted to the natural water [41]. The presence of its residues in aquatic environment has caused increasingly environmental and ecological problems [42-43] and it even shows direct toxicity to certain aquatic microorganisms [44]. Therefore, it is significantly necessary to monitor the residual chloramphenicol in water environment. There are various reported methods for the determination of CAP such as dispersive liquid-liquid microextraction [45], solidphase extraction [46], aqueous two-phase extraction [47] et al. These methods obtained expected results in the quantitative task; but they also have their own shortcomings as described above. More novel, rapid and accurate pretreatment methods are urgently required for the analysis of CAP. 
Based on the above, a novel organic magnetic ionic liquid based on guanidinium cation was synthesized in this study, and then a new method of magnetic ionic liquid aqueous two-phase system (MILATPs) coupled with high-performance liquid chromatography (HPLC) was established to separate effectively and determine trace amount of CAP in water environment. As the result, magnetic separation technique, organic solvent free system and rapid extraction were successfully combined together in this analytical method for the first time. In this work, extraction equilibrium was almost immediately achieved when aqueous two-phase phenomenon occurred and a simple external magnetic field was applied to assist phase separation. Experimental investigations were conducted to optimize different variables in this novel pretreatment process and satisfactory recovery was obtained under optimal conditions. Finally, the method was successfully applied for the determination of CAP in environmental water samples. Hopefully, this novel method can be referenced to monitor trace amount of other antibiotics in water environment.

\section{Experimental}

\subsection{Chemicals and materials}

All reagents used were of analytical grade or above. 4-Hydroxy-2,2,6,6-tetramethyl piperidine 1-oxyl free radical (4-OH-tempo) and 1,1,3,3-tetramethyl-guanidine were purchased from Best Reagent Co., Ltd. (Chengdu, China). Anhydrous potassium phosphate was supplied by Guangfu Superfine Chemical Industry Institute (Tianjin, China). Moreover, chlorosulfonic acid was obtained from Aike Chemical Reagent Co. Ltd. (Chengdu, China). All the other used chemicals and reagents were supplied by Kelong Chemical Co. Ltd. (Chengdu, China). Stock solution I of CAP with 
concentration of $300 \mu \mathrm{g} \mathrm{mL} \mathrm{m}^{-1}$ was prepared by dissolving proper amount of CAP into volumetric flask and then diluting to $50 \mathrm{~mL}$ with deionized water, and the latter was obtained from ultra-pure water purification system $(0.4 \mathrm{~mm}$ filter $)$ manufactured by Millipore Co., Ltd. (Bedford, MA, USA). Stock solution II of CAP was prepared by diluting stock solution I in a $50 \mathrm{~mL}$ volumetric flask to obtain a concentration of 10 $\mu \mathrm{g} \mathrm{mL}^{-1}$. Then they were sealed and stored in a refrigerator until use. Working solutions were prepared weekly by appropriate dilution of stock solution II.

\subsection{Apparatus}

A P1201 high performance liquid chromatography system (Elite, Dalian, China) consisting of UV1201 UV-Vis detector and EC2006 chromatography workstation was used for the analysis and quantitation of CAP. A Welchrom C18 column $(4.6 \times 250$ $\mathrm{mm}, 5 \mu \mathrm{m})$ was used as the HPLC analytical column. The $\mathrm{pH}$ measurement was carried out with a PHS-25 pH-meter (Leici Instrument Factory, Shanghai, China). FTIR spectra of MILs were recorded on a Spectrum Two L1600300 infrared spectrometer (Perkin Elmer, Fremont, USA). Electrospray ionization mass spectra (ESI-MS) were collected on Amazon SL mass spectrometer (Bruker Daltonics, Bremen, Germany) and data were processed by Bruker Compass Data Analysis 4.0. Thermogravimetric (TG) analysis was performed on a HTG-2 Microcomputer Differential Thermal Balance (Hengjiu Scientific Instrument Factory, Beijing, China). The magnetic properties of MIL were investigated by a vibration sample magnetometer (VSM) using MPMS SQUID system (Quantum Design, San Diego, USA). Mass value was determined by a FA/JA type electronic analytical balance (uncertainty of $\pm 0.0001 \mathrm{~g}$ ). A CY20A water thermostat (Boxun Industry \& Commerce Co. Ltd., Shanghai, China) with an uncertainty of $\pm 0.05 \mathrm{~K}$ was applied for keeping 
constant temperature of all the tested system.

\subsection{Synthesis of organic magnetic ionic liquid}

\subsubsection{Synthesis of $\mathrm{H}\left[\mathrm{TEMPO}-\mathrm{OSO}_{3}\right]$}

$\mathrm{H}\left[\mathrm{TEMPO}-\mathrm{OSO}_{3}\right]$ was synthesized according to the procedure of previous literature [48]. Certain amount of 4-hydroxyl-tempo was dissolved in dichloromethane at $0 \sim 5^{\circ} \mathrm{C}$, equal molar of chlorosulfonic acid was added dropwisely. The mixture was continuously stirred for $12 \mathrm{~h}$ at room temperature. After reaction, the product was washed with dichloromethane for 3 times, dried by a DZF-6050 drying oven (Yingyuyuhua Instrument Factory, Gongyi, China) under vacuum at $45^{\circ} \mathrm{C}$ and then dissolved in ethanol, successively.

\subsubsection{Synthesis of [TMG][TEMPO-OSO 3$]$}

$[\mathrm{TMG}]\left[\mathrm{TEMPO}^{\left.-\mathrm{OSO}_{3}\right]}\right.$ was prepared by simple neutralization of H[TEMPO$\left.\mathrm{OSO}_{3}\right]$ with equimolar of 1,1,3,3-tetramethylguanidine in ethanol or acetone. The product was washed with toluene for 3 times and dried under vacuum at $45^{\circ} \mathrm{C}$. The final product was dark-reddish viscous liquid with yield of $91.8 \%$. The MIL was characterized by FT-IR spectra and ESI-MS, its structural formula was shown in Fig. 1.

\subsection{Preparation and measurement of ATPs}

The binodal curves were determined by the cloud point method [49] in a jacketed glass vessel containing a magnetic stirrer at constant temperature and atmospheric pressure. The vessel was immersed in a jacketed glass vessel using water thermostat to keep constant temperature in extrinsic cycle pattern. An IL solution of known mass fraction was taken into the vessel with vigorous stirring for $15 \mathrm{~min}$. Then a salt solution of known mass fraction was added dropwise to the vessel with an interval of 
5 min between every two drops. When the mixture turned cloudy, the stirring was stopped and the two phases began to appear clearly. At the end of ATPs formation, the composition of the mixture for each point on the binodal curve was weighed, noted and calculated by mass. Afterwards, a certain amount of water was added dropwisely to produce a clear one-phase system. The system was then kept stirring at constant temperature for another $15 \mathrm{~min}$. Repetitive operation was kept on to obtain the phase equilibrium line. Finally, the equilibrium curves data were correlated by the Merchuk equation [50] as eq.(1):

$$
w_{1}=\operatorname{aexp}\left(\mathrm{b} w_{2}^{0.5}-c w_{2}^{3}\right)
$$

Where $w_{1}$ and $w_{2}$ are the IL and salt weight percentages, respectively; $\mathrm{a}, \mathrm{b}$ and $\mathrm{c}$ are three constants obtained by regression of the experimental data.

For the determination of each tie-line (TL), a ternary mixture composed of salt+water $+\mathrm{IL}$ at the biphasic region was gravimetrically prepared. The system was stirred and left to equilibrate, and then a complete separation of two phases was obtained. The phases were separately weighed and noted. Each TL was then calculated according to eq.(2-5) proposed by Merchuk [50] et al.

$$
\begin{gathered}
w_{1}^{t}=a \exp \left(b\left(w_{2}^{t}\right)^{0.5}-c\left(w_{2}^{t}\right)^{3}\right) \\
w_{1}^{b}=a \exp \left(b\left(w_{2}^{b}\right)^{0.5}-c\left(w_{2}^{b}\right)^{3}\right) \\
w_{1}^{t}=w_{1}^{m} / \alpha-w_{1}^{b}(1-\alpha) / \alpha \\
w_{2}^{t}=w_{2}^{m} / \alpha-w_{2}^{b}(1-\alpha) / \alpha
\end{gathered}
$$


Where the superscripts of $t, b$ and $m$ designate the top phase, the bottom phase and the mixture, respectively; $\alpha$ is the ratio of the mass of the top phase and the total mass of the mixture. The tie-line length (TLL) corresponds to the distance between the points of the bottom phase composition to that of the top phase composition in the binodal curve.

\subsection{Extraction procedure}

Working solution was prepared by appropriate dilution of stock solution 2 and adjusted to a certain $\mathrm{pH}$ value by phosphoric acid or ammonium hydroxide. A schematic representation of extraction procedure is shown in Fig.2. A certain amount of IL and working solution were successively weighed in a $10 \mathrm{~mL}$ centrifugal tube with the mass accuracy guaranteed by a syringe. Afterwards, complete dissolution of IL was achieved. On the other hand, anhydrous potassium phosphate was placed in thermostatic water bath beforehand to reach the certain required temperature, which could avoid the change of ATPs temperature during salt addition. Then appropriate amount of anhydrous potassium phosphate was added and dissolved in the system at the certain temperature. A turbid or cloudy solution was easily formed without any additional treatment. During this stage, CAP was immediately extracted into the ILrich droplet. Then a strong $\mathrm{NdFeB}$ magnet was used to separate the extraction phase. The magnetic ionic liquid droplet could be attracted by the magnet, and moved gently towards the magnet; then two phases were separated more easily. Because the new MIL has strong magnetism, the complete collection of its dispersing droplets can be achieved within $1 \mathrm{~min}$. Afterwards, the upper IL phase volume was accurately measured by a syringe with an uncertainty of $\pm 0.5 \mu \mathrm{L}$. The mass of the upper IL phase was accurately weighed by an analytical balance with an uncertainty of $\pm 0.0001 \mathrm{~g}$. 
Then $20 \mu \mathrm{L}$ of ionic liquid phase was directly injected into HPLC for quantitative analysis.

\subsection{HPLC analysis and determination of the partition parameters of CAP}

The optimal HPLC condition on the C18 column was determined as the mobile phase of water-acetonitrile $(82: 18, \mathrm{~V} / \mathrm{V})$ at a flow rate of $2.0 \mathrm{~mL} \mathrm{~min}{ }^{-1}$ with $20 \mu \mathrm{L}$ injection volume. The column effluent was monitored at $278 \mathrm{~nm}$, which was the maximum UV absorption wavelength of CAP. Extraction recovery (ER) of CAP was calculated according to eq. (6).

$$
\operatorname{ER}(\%)=v_{I L} \times c_{I L} / m_{s}
$$

Where $v_{I L}(\mathrm{~mL})$ and $C_{I L}\left(\mathrm{~g} \mathrm{~mL}^{-1}\right)$ are the volume of IL-rich phase and concentration of CAP in IL-rich phase, respectively; $m_{s}(\mathrm{~g})$ is the amount of CAP added.

All of CAP preconcentration experiments were performed 3 times repeatedly and their standard deviations (SD) were calculated as eq. (7).

$$
S D=\left(\sum_{i=1}^{N}\left(w_{i}-w_{\text {ave }}\right)^{2} / N\right)^{0.5}
$$

Where ${ }^{w_{i}}$ and ${ }^{w_{\text {ave }}}$ are experimental value each time and the average value, respectively. $N$ represents the repeated experimental times $(N=3)$.

Mass ratio $(\mathrm{R})$ was defined as the mass ratio of the bottom phase $\left(m_{b}\right)$ to top phase $\left(m_{t}\right)$. The preconcentration factor (PF) was defined as the ratio of concentration of CAP in IL-rich phase to initial concentration of CAP in the sample solution. 


\section{Results and discussion}

\subsection{Characterization of MIL}

The structure of new MIL has been identified via FT-IR spectra and ESI-MS as shown in Fig.3a and Fig.3c-3d. In FT-IR spectra, $3355 \mathrm{~cm}^{-1}$ is the N-H stretching vibration; $2976 \mathrm{~cm}^{-1}$ and $2934 \mathrm{~cm}^{-1}$ attribute to the methyl and methylene saturated C$\mathrm{H}$ stretching vibration in the anion; $1607 \mathrm{~cm}^{-1}$ is the $\mathrm{C}=\mathrm{N}$ stretching vibration; 1568 $\mathrm{cm}^{-1}$ is the N-H in-plane bending vibration; $1364 \mathrm{~cm}^{-1}, 1411 \mathrm{~cm}^{-1}$ and $1464 \mathrm{~cm}^{-1}$ are the methyl and methylene $\mathrm{C}-\mathrm{H}$ in-plane bending vibration; $1215 \mathrm{~cm}^{-1}$ is the $\mathrm{C}-\mathrm{N}$ stretching vibration; $1062 \mathrm{~cm}^{-1}$ is the $\mathrm{N}-\mathrm{O}$ stretching vibration; $793 \mathrm{~cm}^{-1}$ is the $\mathrm{N}-\mathrm{H}$ out-of-plane bending vibration. In ESI-MS spectrum, there existed fragment ions with $\mathrm{m} / \mathrm{e} 116\left(\mathrm{C}_{5} \mathrm{H}_{14} \mathrm{~N}_{3}^{+}\right)$in positive ion mode and $\mathrm{m} / \mathrm{e} 251\left(\mathrm{C}_{9} \mathrm{H}_{17} \mathrm{NO}_{5} \mathrm{~S}^{-}\right)$in negative ion mode. Above data can prove the structure of new MIL after comparison with the recent study [23]. Thermogravimetric (TG) curve of novel [TMG][TEMPO-OSO 3 ] is shown in Fig.3b. It can be concluded that this MIL was very stable when temperature was lower than $140^{\circ} \mathrm{C}$. So this novel MIL is suitable for the following analytical method.

\subsection{Study of the magnetization and susceptibility of MIL}

The magnetic property of MIL was investigated by a vibration sample magnetometer (VSM) using MPMS SQUID system (Quantum Design, America). As shown in Fig.4, the magnetic susceptibility at temperature of $298 \mathrm{~K}$ and $5 \mathrm{~K}$ revealed linear growth with the increasing intensity of applied magnetic field. In addition, static susceptibility measurement against the temperature under an applied field of $2 \mathrm{~T}$ was also investigated. It was found [TMG][TEMPO-OSO 3$]$ exhibited a weak 
paramagnetic property when temperature was above $150 \mathrm{~K}$. Furthermore, when temperature was lowered, its magnetization rose sharply to a very strong magnetic susceptibility value as high as $855 \mathrm{emu} \mathrm{mol}^{-1}$, which is about 80 times of the value at room temperature $(298 \mathrm{~K})$. This phenomenon reveals great potential for its magnetic application under low temperature. Additionally, the magnetic response of ionic liquid droplets to a neodymium magnet in an aqueous two phase system was demonstrated in Fig.5. It can be found that the droplets could be attracted by the magnet strongly and move with it. As a result, it is beneficial for enhancing the efficiency of phase separation.

\subsection{Phase behavior of magnetic ILATPs}

\subsubsection{Effect of the property of salts on phase separation}

The type of salts can play a very key role in the previous reports of ATPs, so a series of inorganic salts were firstly tested for the formation of the new MILATPs with [TMG][TEMPO-OSO 3 . Results demonstrate that MILATPs can form by adding appropriate amount of some specific salts, such as $\mathrm{K}_{3} \mathrm{PO}_{4}, \mathrm{~K}_{2} \mathrm{CO}_{3}, \mathrm{Na}_{2} \mathrm{CO}_{3}, \mathrm{Na}_{2} \mathrm{SO}_{4}$ or $\left(\mathrm{NH}_{4}\right)_{2} \mathrm{SO}_{4}$. When other salts or some bases were selected, such as $\mathrm{NaCl}, \mathrm{KCl}$, $\mathrm{K}_{2} \mathrm{HPO}_{4}, \mathrm{KOH}$ or $\mathrm{NaOH}$, they cannot drive MIL aqueous solution to separate into two phases. Fig.6 shows the phase diagrams of MIL aqueous two-phase systems with different inorganic salts at $298.15 \mathrm{~K}$. It can be seen that experimental data of IL$\left(\mathrm{NH}_{4}\right)_{2} \mathrm{SO}_{4}$ system do not fit very well with the calculated values. This result attributes to the relatively poor phase-separating ability of $\left(\mathrm{NH}_{4}\right)_{2} \mathrm{SO}_{4}$. As for those systems containing $\mathrm{Na}_{2} \mathrm{CO}_{3}$ and $\mathrm{Na}_{2} \mathrm{SO}_{4}$, the two salts have relatively low solubility in water and low salting-out ability. Consequently, MIL cannot be easily salted out if its amount is small. Considering the essence of microextraction, IL- $\mathrm{K}_{3} \mathrm{PO}_{4}$ and IL- 
$\mathrm{K}_{2} \mathrm{CO}_{3}$ are much more suitable for preconcentration of CAP than other salts. Further comparison between these two systems was carried out by the experimental tie-lines. In IL- $\mathrm{K}_{3} \mathrm{PO}_{4}$ system, tie-line data reveal that when top phase is composed of nearly $80 \%$ of IL, the bottom phase only has $0.2 \%$ of IL dissolved. However, in IL- $\mathrm{K}_{2} \mathrm{CO}_{3}$ system, there still exists $2.8 \%$ of IL dissolved in the bottom phase when top phase is almost $100 \%$ composed of IL which is a saturation condition. Although both of them are fine salting-out agent, $\mathrm{K}_{3} \mathrm{PO}_{4}$ demonstrates more ideal performance and is more suitable in the microextraction application.

\subsubsection{Effect of temperature}

According to the result of the above section, $\mathrm{K}_{3} \mathrm{PO}_{4}$ was selected in the MILATPs to further investigate the effect of temperature on the phase separation ability. The thermal influences are illustrated in Fig.7. It is obvious that, when the mass fraction of anhydrous potassium phosphate is less than $19 \%$, the phase-forming ability of magnetic ionic liquid will decrease with temperature rising. But there seems to be not much difference of phase behavior when $\mathrm{K}_{3} \mathrm{PO}_{4}$ content in the system becomes higher. It can be concluded that interaction of ionic liquid with water will increase when temperature is higher and their mutual solubility is enhanced. Although all temperatures could satisfy the conditions of subsequent study of CAP pretreatment, room temperature $(298.15 \mathrm{~K})$ was chosen for operation simplicity and energy saving.

\subsection{Separation and concentration behaviors of CAP in MILATPs}

\subsubsection{Effect of the concentration of $\mathrm{K}_{3} \mathrm{PO}_{4}$}

Effect of the concentration of $\mathrm{K}_{3} \mathrm{PO}_{4}$ on extraction recovery and preconcentration factor of CAP as well as mass ratio of the microextraction system was discussed in detail. The concentration of working solution of CAP was $1000 \mathrm{ng} \mathrm{mL}^{-1}$. As shown in 
Fig.8, with the increasing of $\mathrm{K}_{3} \mathrm{PO}_{4}$ concentration, the recovery of CAP can reach up to $99.82 \%$. When $\mathrm{K}_{3} \mathrm{PO}_{4}$ is more than $4.08 \mathrm{~g}(36.92 \%)$, extraction recovery keeps relatively steady. It is regarded as a strong salting-out salt for its relatively higher hydrophilicity, and the MIL tends to separate from the system to form another phase as the amount of salt is increased. Moreover, CAP has a much better affinity with $[\mathrm{TMG}]\left[\mathrm{TEMPO}-\mathrm{OSO}_{3}\right]$ than water probably due to the strong hydrogen bonds between CAP and IL. As a result, the salt will promote CAP to transfer into the ILrich phase. The more salt was added, the more IL was separated out to form a new phase and the more amount of CAP was extracted to IL-rich phase. But when the salt is sufficient enough for the complete extraction of CAP, more addition of salt is not necessary. So $\mathrm{K}_{3} \mathrm{PO}_{4}$ with the amount of $4.08 \mathrm{~g}$ is selected for further investigation.

\subsubsection{Effect of initial mass of [TMG][TEMPO-OSO 3$]$}

In this section, the effect of initial mass of magnetic ionic liquid in the new MILATPs for pretreatment of CAP was discussed. As demonstrated in Fig.9, with the increase of the amount of IL, the extraction efficiency of CAP can be improved significantly while the preconcentration factor rises slightly and then drops significantly. Extraction efficiency is obviously enhanced with initial increase of MIL mass and then reaches equilibrium with further addition of MIL. Generally, both high extraction recovery and ideal pretreatment factor are crucial for the determination of trace amount of target component. Considering these two aspects, $0.05 \mathrm{~g}$ of IL was appropriate with extraction recovery of $99.82 \%$ and preconcentration factor of 146 for CAP.

\subsubsection{Effect of pH of sample solution}

Experiments were performed by changing the $\mathrm{pH}$ value of sample solution from 1 
to 13 by adding appropriate amounts of phosphoric acid and ammonium hydroxide. Related effects of $\mathrm{pH}$ on the recovery of CAP and preconcentration factor as well as mass ratio are shown in Fig.10. According to the experimental result, it was found extraction recovery of CAP decreased sharply when $\mathrm{pH}>7$. The reason was that CAP could be gradually decomposed if it was preserved in alkaline condition for a certain time. On the contrary, when $\mathrm{pH} \leq 7$, the level of CAP recovery keeps relatively steady and reaches the highest value at $\mathrm{pH}=3$. In the extraction process applied in our study, although the salt-rich phase was alkaline after dissolution of $\mathrm{K}_{3} \mathrm{PO}_{4}$, CAP was almost immediately extracted into the fine droplets of MIL. In such a short time, $\mathrm{K}_{3} \mathrm{PO}_{4}$ and CAP could hardly coexist in the same phase. Meanwhile, the decomposition of CAP usually needs more time and enough alkaline. Such short exposure to alkaline cannot change its molecular structure, which should attribute to the rapid phase separation. To avoid possible decomposition, $\mathrm{pH}$ value of original sample solution can be determined to be 3 as the optimal one.

\subsection{Method validation and comparison}

A new analytical method of MILATPs coupled with HPLC was developed for quantitative determination of trace amount of CAP in environmental water. Under the optimum conditions, the calibration curve was performed by applying standard CAP solution at nine different concentrations with three replications for each one in the MILATPs in the range of $10 \sim 2500 \mathrm{ng} \mathrm{mL} \mathrm{m}^{-1}$. The obtained linear regression equation was $y=1.5332 x+1.6386\left(\mathrm{R}^{2}=0.9995\right)$, where $y$ is peak area of CAP and $x$ represents the concentration (ng mL-1) of CAP. Good linear range was achieved from $12.25 \mathrm{ng} \mathrm{mL}^{-1}$ to $2200 \mathrm{ng} \mathrm{mL} \mathrm{m}^{-1}$.

The limit of detection (LOD) and the limit of quantification (LOQ) based on $3 \mathrm{~S} / \mathrm{m}$ 
and $10 \mathrm{~S}_{\mathrm{b}} / \mathrm{m}$ (where $\mathrm{S}_{\mathrm{b}}$ is standard deviation of the blank signals and $\mathrm{m}$ is the slope of calibration curve after pretreatment) were determined as $0.14 \mathrm{ng} \mathrm{mL}^{-1}$ and $0.46 \mathrm{ng}$ $\mathrm{mL}^{-1}$, respectively. This reveals a good sensitivity for the presented method.

Finally, defined as the relative standard deviation (RSD\%), the intra-day and interday precision were determined with several replications at the concentration level of $220 \mathrm{ng} \mathrm{mL} \mathrm{m}^{-1}$ of each analyte. They were analyzed by independent pretreatment of samples using the proposed method six times in a day or in different six days with achieved $\mathrm{RSD} \%$ as $2.42 \%$ for intra-day precision and $4.45 \%$ for inter-day precision, respectively. Both of them were acceptable. Recoveries were all satisfactorily in the range of $90 \% \sim 110 \%$ for this method. The preconcentration factor (PF) of this novel method was calculated as 147.2 , which was much higher than those in reported ATPs literatures $[22,47]$. As for other newer methods of extraction, the extractant droplet in single drop microextraction (SDME) is easily disturbed by water phase under highspeed stirring, which will result in the lower repeatability and limit its use [33], then dispersive liquid-liquid microextraction (DLLME) was developed as a simple and rapid pre-concentration method for the analysis of some organic compounds in water. But tedious centrifugation operation [6] and the use of organic solvent (e.g. ethyl acetate, $n$-hexane and so on) cannot be avoided [35-36]. New methods with magnetic nanoparticles applied in the DLLME system [11] can realize rapid separation, but the self-made nanoparticles cannot be easily obtained and inevitable desorption procedure is time-consuming. The earlier ATPs extraction method has avoided the use of volatile organic solvent successfully, such as in the analysis for human urine [51]. However, entire phase separation was difficult and the researchers had to employ extra postprocessing before analysis. In this study, novel MILATPs method is fast and easy to 
operate without centrifugation or desorption procedure, which enhances sample treatment efficiency greatly under greener condition. Compared with above methods, this is the first time to combine the advantages of organic solvent-free extraction, rapid preconcentration and magnetic separation simultaneously.

\subsection{Analysis of real samples}

In order to investigate the enrichment performance for real samples, the proposed method was applied for the pretreatment and determination of CAP in Jiangan river, Mingyuan lake and tap water of our laboratory, all located in Jiangan campus of Sichuan University, Shuangliu county, Chengdu city, China. All of the selected samples were centrifuged at $4000 \mathrm{rpm}$ for $5 \mathrm{~min}$ and after that the supernatant was collected, then $\mathrm{pH}$ was adjusted for analysis. Spiked recovery experiments were performed by adding a very small amount of CAP working solution into the collected sample solution to obtain the required spiked concentration followed by $\mathrm{pH}$ adjustment. All the above environmental samples were filtered through microporous membrane with a pore size of $0.22 \mu \mathrm{m}$ before HPLC analysis. The CAP analysis was carried out three times for each sample following the procedure described above. The results are listed in Table 1. The accuracy of the proposed method was evaluated by analysis of spiked real samples with the extraction recovery (ER, \%) acquired from eq. (8):

$$
E R(\%)=\left(C_{\text {found }}-C_{\text {real }}\right) / C_{\text {added }} \times 100 \%
$$

Where $\mathrm{C}_{\text {found }}, \mathrm{C}_{\text {real }}$ and $\mathrm{C}_{\text {added }}$ are the total detected concentration of CAP after addition of known amount of standard analyte into the real sample, the concentration of analyte in real environmental sample and the concentration of the known added concentration in real sample, respectively. As shown in Table 1, good recovery of 
analyte was obtained in the range of $94.6 \% \sim 99.72 \%$ with RSD of $1.97 \% \sim 4.35 \%$. Additionally, it reveals that the present novel method has a good reliability and satisfactory reproducibility for the determination of trace amount of CAP in environmental waters. HPLC chromatograms with spiked concentration of $50 \mathrm{ng} \mathrm{mL}{ }^{-1}$ are shown in Fig.11, which exhibits a good base separation of the peak of CAP with others in such a complex analyte. In summary, the proposed method in this work can be satisfactorily applied for determination of trace amount of CAP in the environmental samples.

\section{Conclusion}

A unique aqueous two-phase system based on new magnetic ionic liquid with special property was developed, which was used to establish a novel analytical method with high performance liquid chromatography to separate effectively and determine trace amount of chloramphenicol in environmental waters. The advantages of magnetic separation, no use of organic solvent and rapid extraction were combined together in this MILATPs-HPLC method for the first time. An external magnetic field was applied for phase separation and it successfully enhanced the pretreatment efficiency. Extraction procedure could be almost immediately completed without any further operation such as vortex shaking. Preconcentration factor of 147.2 was obtained, which shows a significant enrichment effect. Additionally, good linear range of $12.25 \mathrm{ng} \mathrm{mL}^{-1} \sim 2200 \mathrm{ng} \mathrm{mL}^{-1}$ and fine precision $\mathrm{RSD}^{\circ} \%$ (2.42\% for intra-day, $4.45 \%$ for inter-day) as well as low value of limit of detection and limit of quantitation ( 0.14 $\mathrm{ng} \mathrm{mL} \mathrm{mL}^{-1}$ and $0.42 \mathrm{ng} \mathrm{mL}^{-1}$, respectively) were acquired by this method. Good recovery of $90 \% \sim 110 \%$ for chloramphenicol was also achieved in the analysis of real 
environmental samples. In a word, the novel magnetic aqueous two-phase system is showing great potential and is expected to promote new development in the field of extraction, separation and pretreatment of various samples.

\section{Acknowledgments}

Preparation of this paper was supported by National Science Foundation of China (No. 81373284) and 2013 scientific research foundation of Sichuan University for Outstanding Young Scholars (No.2082604184184).

\section{References}

[1] Z.M. Liu, X.H. Zang, W.H. Liu, C. Wang, and Z. Wang, Novel method for the determination of five carbamate pesticides in water samples by dispersive liquidliquid microextraction combined with high performance liquid chromatography. Chinese Chem. Lett. 20 (2009) 213-216.

[2] M.T. Galceran, P. Pais, and L. Puignou, Isolation by solid-phase extraction and liquid chromatographic determination of mutagenic amines in beef extracts. J. Chromatogr. A. 719 (1996) 203-212.

[3] Y.S. Gu, I.S. Kim, J.K. Ahn, D.C. Park, D.M. Yeum, C.I. Ji, and S.B. Kim, Mutagenic and carcinogenic heterocyclic amines as affected by muscle types/skin and cooking in pan-roasted mackerel. Mutat. Res-Gen.Tox. En. 515 (2002) 189-195.

[4] C. Yu, J. Han, Y. Wang, Y. Yan, S. Hu, Y. Li, and C. Ma, Ionic liquid/ammonium sulfate aqueous two-phase system coupled with HPLC extraction of sulfadimidine in real environmental water samples. Chromatographia. 74 (2011) 407-413.

[5] V. Ghasemzadeh-Mohammadi, A. Mohammadi, M. Hashemi, R. Khaksar, and P. Haratian, Microwave-assisted extraction and dispersive liquid-liquid microextraction 
followed by gas chromatography-mass spectrometry for isolation and determination of polycyclic aromatic hydrocarbons in smoked fish. J. Chromatogr. A. 1237 (2012) $30-36$.

[6] M. Rezaee, Y. Assadi, M.M. Hosseini, E. Aghaee, F. Ahmadi, and S. Berijani, Determination of organic compounds in water using dispersive liquid-liquid microextraction. J. Chromatogr. A. 1116 (2006) 1-9.

[7] M. Kamankesh, A. Mohammadi, H. Hosseini, and Z.M. Tehrani, Rapid determination of polycyclic aromatic hydrocarbons in grilled meat using microwaveassisted extraction and dispersive liquid-liquid microextraction coupled to gas chromatography-mass spectrometry. Meat Sci. 103 (2015) 61-67.

[8] M. Madani-Tonekaboni, M. Kamankesh, and A. Mohammadi, Determination of furfural and hydroxymethyl furfural from baby formula using dispersive liquid-liquid microextraction coupled with high performance liquid chromatography and method optimization by response surface methodology. J. Food Compos. Anal. 40 (2015) 1-7. [9] A. Mohammadi, V. Ghasemzadeh-Mohammadi, P. Haratian, R. Khaksar, and M. Chaichi, Determination of polycyclic aromatic hydrocarbons in smoked fish samples by a new microextraction technique and method optimisation using response surface methodology. Food Chem. 141 (2013) 2459-2465.

[10] Y. Nojavan, M. Kamankesh, F. Shahraz, M. Hashemi, and A. Mohammadi, Ion pair-based dispersive liquid-liquid microextraction followed by high performance liquid chromatography as a new method for determining five folate derivatives in foodstuffs. Talanta. 137 (2015) 31-37.

[11] Z. Shi, and H.K. Lee, Dispersive liquid-liquid microextraction coupled with dispersive $\mu$-solid-phase extraction for the fast determination of polycyclic aromatic 
hydrocarbons in environmental water samples. Anal. Chem. 82 (2010) 1540-1545.

[12] P. Albertsson, Partition of cell particles and macromolecules: separation and purification of biomolecules, cell organelles, membranes, and cells in aqueous polymer two-phase systems and their use in biochemical analysis and biotechnology, Wiley New York etc., 1986.

[13] Z. Gu, and C.E. Glatz, Aqueous two-phase extraction for protein recovery from corn extracts. J. Chromatogr. B 845 (2007) 38-50.

[14] B. Mokhtarani, R. Karimzadeh, M.H. Amini, and S.D. Manesh, Partitioning of Ciprofloxacin in aqueous two-phase system of poly (ethylene glycol) and sodium sulphate. Biochem. Eng. J. 38 (2008) 241-247.

[15] M. Shibukawa, N. Nakayama, T. Hayashi, D. Shibuya, Y. Endo, and S. Kawamura, Extraction behaviour of metal ions in aqueous polyethylene glycolsodium sulphate two-phase systems in the presence of iodide and thiocyanate ions. Anal. Chim.Acta. 427 (2001) 293-300.

[16] A. Tong, J. Dong, and L. Li, Aqueous two-phase extraction system of sodium perfluorooctanoate and dodecyltriethylammonium bromide mixture and its application to porphyrins and dyes. Anal. Chim.Acta. 390 (1999) 125-131.

[17] X. Han, and D.W. Armstrong, Ionic liquids in separations. Accounts Chem. Res. 40 (2007) 1079-1086.

[18] X. Chen, A. Sakurazawa, K. Sato, K. Tsunoda, and J. Wang, A Solid-cladding/ liquid-core/liquid-cladding sandwich optical waveguide for the study of dynamic extraction of dye by ionic liquid BmimPF6. Appl. Spectrosc. 66 (2012) 798-802.

[19] C. Yao, T. Li, P. Twu, W.R. Pitner, and J.L. Anderson, Selective extraction of emerging contaminants from water samples by dispersive liquid-liquid 
microextraction using functionalized ionic liquids. J. Chromatogr. A 1218 (2011) $1556-1566$

[20] K.E. Gutowski, G.A. Broker, H.D. Willauer, J.G. Huddleston, R.P. Swatloski, J.D. Holbrey, and R.D. Rogers, Controlling the aqueous miscibility of ionic liquids: aqueous biphasic systems of water-miscible ionic liquids and water-structuring salts for recycle, metathesis, and separations. J. Am. Chem. Soc. 125 (2003) 6632-6633.

[21] Y. Deng, T. Long, D. Zhang, J. Chen, and S. Gan, Phase diagram of [Amim] Cl+ salt aqueous biphasic systems and its application for [Amim] $\mathrm{Cl}$ recovery. J. Chem. Eng. Data. 54 (2009) 2470-2473.

[22] J. Han, Y. Wang, C. Yu, C. Li, Y. Yan, Y. Liu, and L. Wang, Separation, concentration and determination of chloramphenicol in environment and food using an ionic liquid/salt aqueous two-phase flotation system coupled with highperformance liquid chromatography. Anal.Chim.Acta. 685 (2011) 138-145.

[23] T. Yao, S. Yao, D. Tang, L. Jing, D. Wang, and H. Song, Synthesis, magnetism, aqueous-two phase formation and physical properties of novel guanidinium-based magnetic ionic liquids. RSC Advances. 6 (2016) 52898-52904.

[24] H. Qiu, M. Takafuji, X. Liu, S. Jiang, and H. Ihara, Investigation of $\pi-\pi$ and iondipole interactions on 1-allyl-3-butylimidazolium ionic liquid-modified silica stationary phase in reversed-phase liquid chromatography. J. Chromatogr. A. 1217 (2010) 5190-5196.

[25] M. Li, J. Zhang, Y. Li, B. Peng, W. Zhou, and H. Gao, Ionic liquid-linked dual magnetic microextraction: A novel and facile procedure for the determination of pyrethroids in honey samples. Talanta. 107 (2013) 81-87.

[26] E. Yilmaz, and M. Soylak, Ionic liquid-linked dual magnetic microextraction of 
lead (II) from environmental samples prior to its micro-sampling flame atomic absorption spectrometric determination. Talanta. 116 (2013) 882-886.

[27] B. Mallick, B. Balke, C. Felser, and A.V. Mudring, Dysprosium roomtemperature ionic liquids with strong luminescence and response to magnetic fields. Angew. Chem. Int. Edit. 47 (2008) 7635-7638.

[28] M. Li, J. Zhang, Y. Li, B. Peng, W. Zhou, and H. Gao, Ionic liquid-linked dual magnetic microextraction: A novel and facile procedure for the determination of pyrethroids in honey samples. Talanta. 107 (2013) 81-87.

[29] E. Yilmaz, and M. Soylak, Ionic liquid-linked dual magnetic microextraction of lead (II) from environmental samples prior to its micro-sampling flame atomic absorption spectrometric determination. Talanta. 116 (2013) 882-886.

[30] S. Hayashi, and H. Hamaguchi, Discovery of a magnetic ionic liquid [bmim] $\mathrm{FeCl}_{4}$. Chem. Lett. 33 (2004) 1590-1591.

[31] P. Brown, A. Bushmelev, C.P. Butts, J. Cheng, J. Eastoe, I. Grillo, R.K. Heenan, and A.M. Schmidt, Magnetic control over liquid surface properties with responsive surfactants. Angew. Chem. Int. Edit. 51 (2012) 2414-2416.

[32] W. Zhu, P. Wu, L. Yang, Y. Chang, Y. Chao, H. Li, Y. Jiang, W. Jiang, and S. Xun, Pyridinium-based temperature-responsive magnetic ionic liquid for oxidative desulfurization of fuels. Chem. Eng. J. 229 (2013) 250-256.

[33] N. Deng, M. Li, L. Zhao, C. Lu, S.L. de Rooy, and I.M. Warner, Highly efficient extraction of phenolic compounds by use of magnetic room temperature ionic liquids for environmental remediation. J. Hazard. Mater. 192 (2011) 1350-1357.

[34] S.H. Lee, S.H. Ha, C. You, and Y. Koo, Recovery of magnetic ionic liquid [bmim] $\mathrm{FeCl}_{4}$ using electromagnet. Korean J. Chem. Eng. 24 (2007) 436-437. 
[35] Y. Wang, Y. Sun, B. Xu, X. Li, R. Jin, H. Zhang, and D. Song, Magnetic ionic liquid-based dispersive liquid-liquid microextraction for the determination of triazine herbicides in vegetable oils by liquid chromatography. J. Chromatogr. A. 1373 (2014) $9-16$.

[36] A. Beiraghi, M. Shokri, S. Seidi, and B.M. Godajdar, Magnetomotive room temperature dicationic ionic liquid: A new concept toward centrifuge-less dispersive liquid-liquid microextraction. J. Chromatogr. A. 1376 (2015) 1-8.

[37] Y. Yoshida, H. Tanaka, and G. Saito, Organic paramagnetic ionic liquids based on anion containing 2, 2, 6, 6-tetramethyl-1-piperidinyloxyl radical moiety. Chem. Lett. 36 (2007) 1096-1097.

[38] C. Miao, J. Wang, B. Yu, W. Cheng, J. Sun, S. Chanfreau, L. He, and S. Zhang, Synthesis of bimagnetic ionic liquid and application for selective aerobic oxidation of aromatic alcohols under mild conditions. Chem. Commun. 47 (2011) 2697-2699.

[39] D. Gottlieb, and M. Legator, The growth and metabolic behavior of Streptomyces venezuelae in liquid culture. Mycologia. 45 (1953) 507-515.

[40] M.J. Bogusz, H. Hassan, E. Al-Enazi, Z. Ibrahim, and M. Al-Tufail, Rapid determination of chloramphenicol and its glucuronide in food products by liquid chromatography-electrospray negative ionization tandem mass spectrometry. J. Chromatogr. B. 807 (2004) 343-356.

[41] T. Heberer, Occurrence, fate, and removal of pharmaceutical residues in the aquatic environment: a review of recent research data. Toxicol. Lett. 131 (2002) 5-17.

[42] B.E. Erickson, Analyzing the ignored environmental contaminants. Environ. Sci. Technol. 36 (2002) 140A-145A.

[43] C.G. Daughton, Non-regulated water contaminants: emerging research. 
Environmental Impact Assessment Review 24 (2004) 711-732.

[44] L. Wollenberger, B. Halling-Sørensen, and K.O. Kusk, Acute and chronic toxicity of veterinary antibiotics to Daphnia magna. Chemosphere. 40 (2000) 723-730.

[45] H. Chen, H. Chen, J. Ying, J. Huang, and L. Liao, Dispersive liquid-liquid microextraction followed by high-performance liquid chromatography as an efficient and sensitive technique for simultaneous determination of chloramphenicol and thiamphenicol in honey. Anal. Chim. Acta. 632 (2009) 80-85.

[46] H. Kubala-Drincic, D. Bazulic, J. Sapunar-Postruznik, M. Grubelic, and G. Stuhne, Matrix solid-phase dispersion extraction and gas chromatographic determination of chloramphenicol in muscle tissue. J. Agr. Food Chem. 51 (2003) $871-875$

[47] J. Han, Y. Wang, C. Yu, Y. Yan, and X. Xie, Extraction and determination of chloramphenicol in feed water, milk, and honey samples using an ionic liquid/sodium citrate aqueous two-phase system coupled with high-performance liquid chromatography. Anal. Bioanal. Chem. 399 (2011) 1295-1304.

[48] H. Akutsu, J. Yamada, and S. Nakatsuji, Preparation and characterization of novel organic radical anions for organic conductors: TEMPO-NHSO${ }_{3}$-and TEMPO$\mathrm{OSO}_{3}$. Synthetic Met. 120 (2001) 871-872.

[49] B. Y. Zasavsky, Aqueous two-phase partitioning, first ed,, Marcel Decker, New York, 1995.

[50] J.C. Merchuk, B.A. Andrews, and J.A. Asenjo, Aqueous two-phase systems for protein separation: Studies on phase inversion. J. Chromatogr. B 711 (1998) 285-293.

[51] C. He, S. Li, H. Liu, K. Li, and F. Liu, Extraction of testosterone and 
epitestosterone in human urine using aqueous two-phase systems of ionic liquid and salt. J. Chromatogr. A 1082 (2005) 143-149. 


\section{Figure captions}

Fig.1 The structure of new magnetic ionic liquid

Fig.2 Scheme of MILATPs extraction procedure

Fig.3 FT-IR (a), TG curve (b) and ESI-MS (positive mode: c and negative mode: d) of MIL

Fig.4 Magnetic properties of new MIL.

Fig.5 Magnetic response of MIL droplets to a neodymium magnet

Fig.6 Binodal curves of $\mathrm{MIL}+\mathrm{salt}+\mathrm{H}_{2} \mathrm{O}$ at $298.15 \mathrm{~K}$. The pointsare experimental data; the curve is composed of calculated data according to Eq.(1), and the straight line is tie-line.

Fig.7 Binodal curves of $\mathrm{MIL}+\mathrm{K}_{3} \mathrm{PO}_{4}+\mathrm{H}_{2} \mathrm{O}$ at different temperature. The points are experimental data; the curve is composed of calculated data according to Eq.(1).

Fig.8 Effect of the concentration of $\mathrm{K}_{3} \mathrm{PO}_{4}$ on the recovery of CAP with conditions: IL: $0.05 \mathrm{~g}$; working solution: $6.92 \mathrm{~g}$; $\mathrm{pH}$ of sample solution: 7; extraction temperature: $278.15 \mathrm{~K}$; extraction time: immediately after dissolving $\mathrm{K}_{3} \mathrm{PO}_{4}$ (Error bars show the standard deviation of the mean, $n=3$ )

Fig.9 Effect of initial mass of MIL on the recovery of CAP with conditions: $\mathrm{K}_{3} \mathrm{PO}_{4}$ : $4.08 \mathrm{~g}$; working solution: $6.92 \mathrm{~g}$; extraction temperature: $298.15 \mathrm{~K}$; extraction time: immediately after dissolving $\mathrm{K}_{3} \mathrm{PO}_{4}$ (error bars show the standard deviation of the mean, $\mathrm{n}=3$ )

Fig.10 Effect of $\mathrm{pH}$ on the recovery of CAP with conditions: $\mathrm{K}_{3} \mathrm{PO}_{4}: 4.08 \mathrm{~g}$; IL: 0.05 g; working solution: $6.92 \mathrm{~g}$; extraction temperature: $298.15 \mathrm{~K}$; extraction time: immediately after dissolving $\mathrm{K}_{3} \mathrm{PO}_{4}$ (error bars show the standard deviation of the mean, $\mathrm{n}=3$ ) 
Fig.11 HPLC chromatograms of three sample solutions added with $50 \mathrm{ng} \mathrm{mL}^{-1} \mathrm{CAP}$ after MILATPs extraction, including a: Jiangan river water, b: Mingyuan lake water and c: tap water in laboratory. 
Fig.1
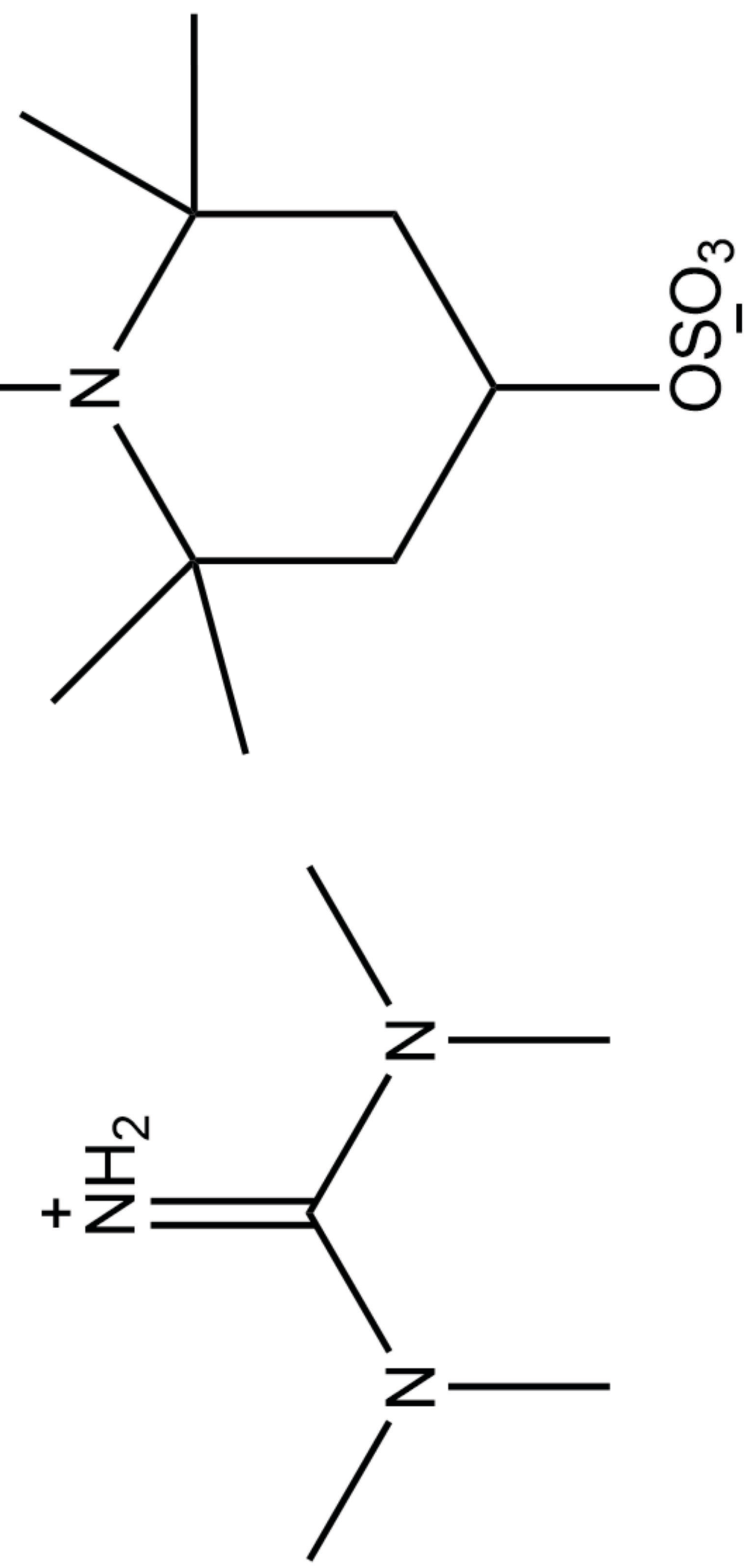


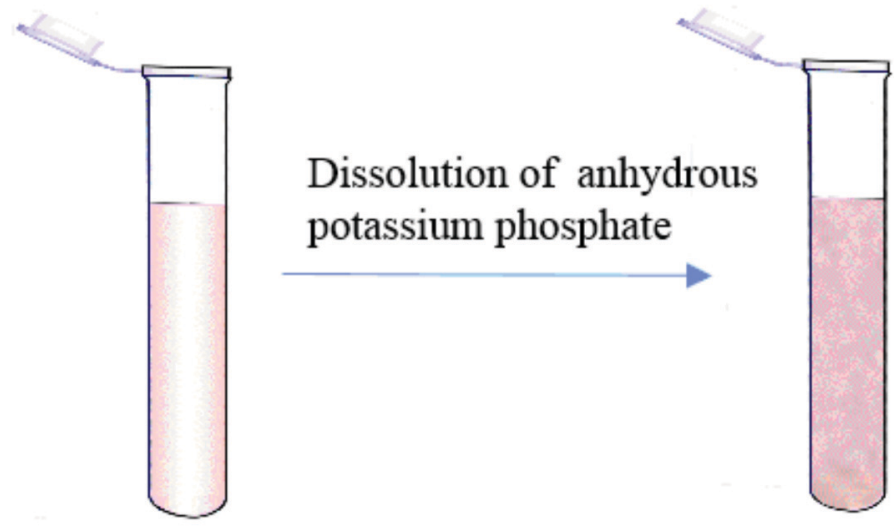

MIL dissolved in CAP containing water sample
Cloudy solution with

MIL salted out

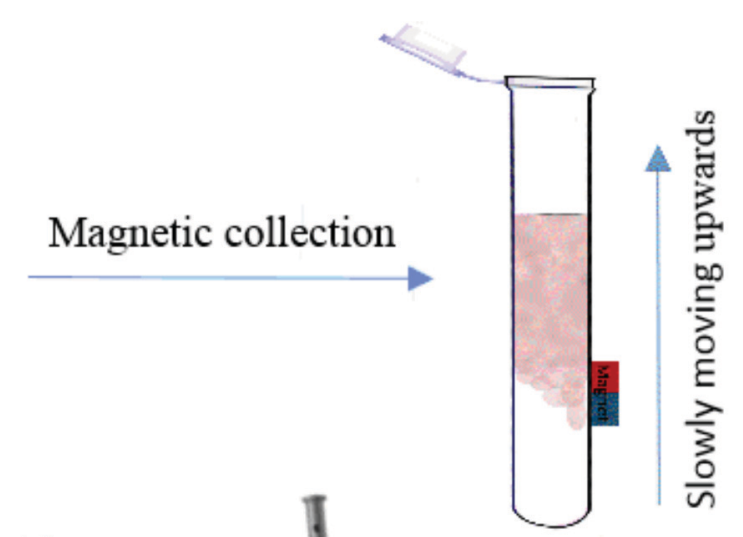

Paramagnetic drops attracted by a magnet

Magnetic collection

Withdrawn into syringe

\section{HPLC analysis}

Paramagnetic drops attracted by a magnet 


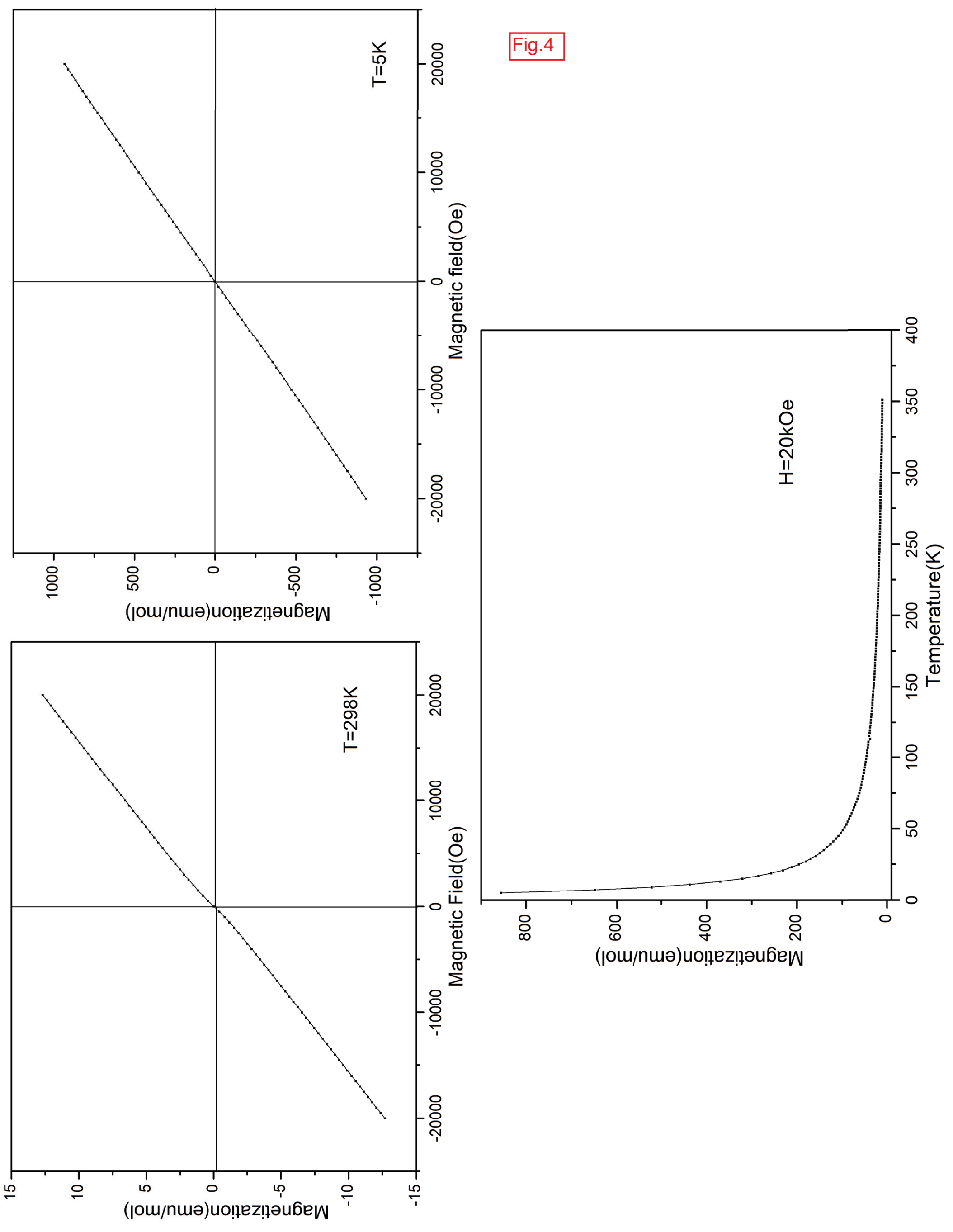


Fig.5

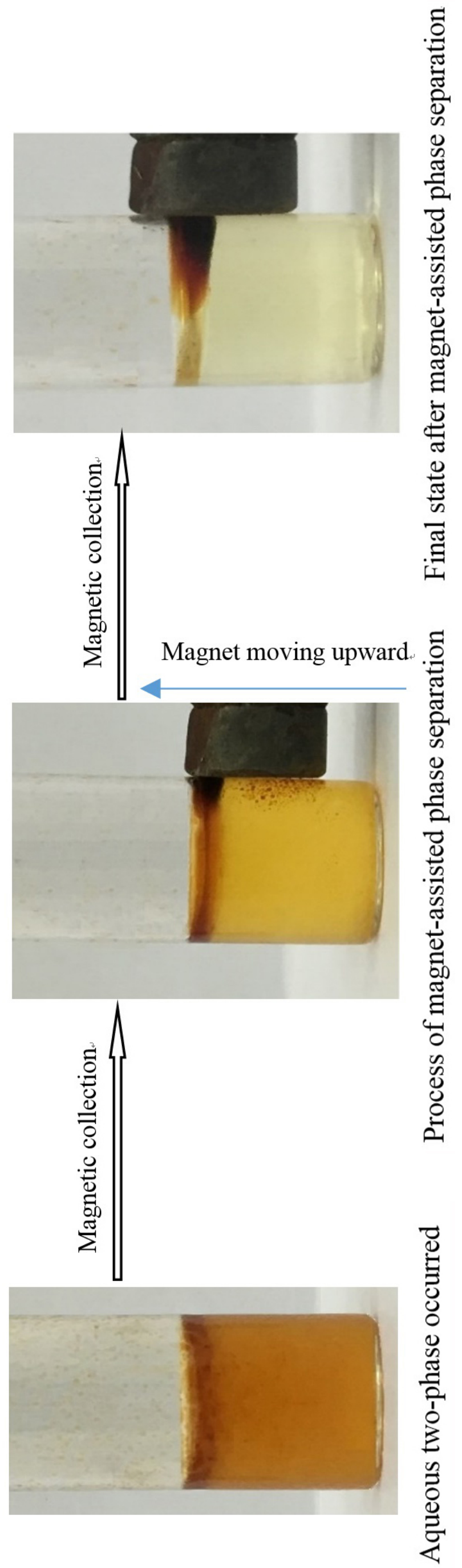


Fig.6

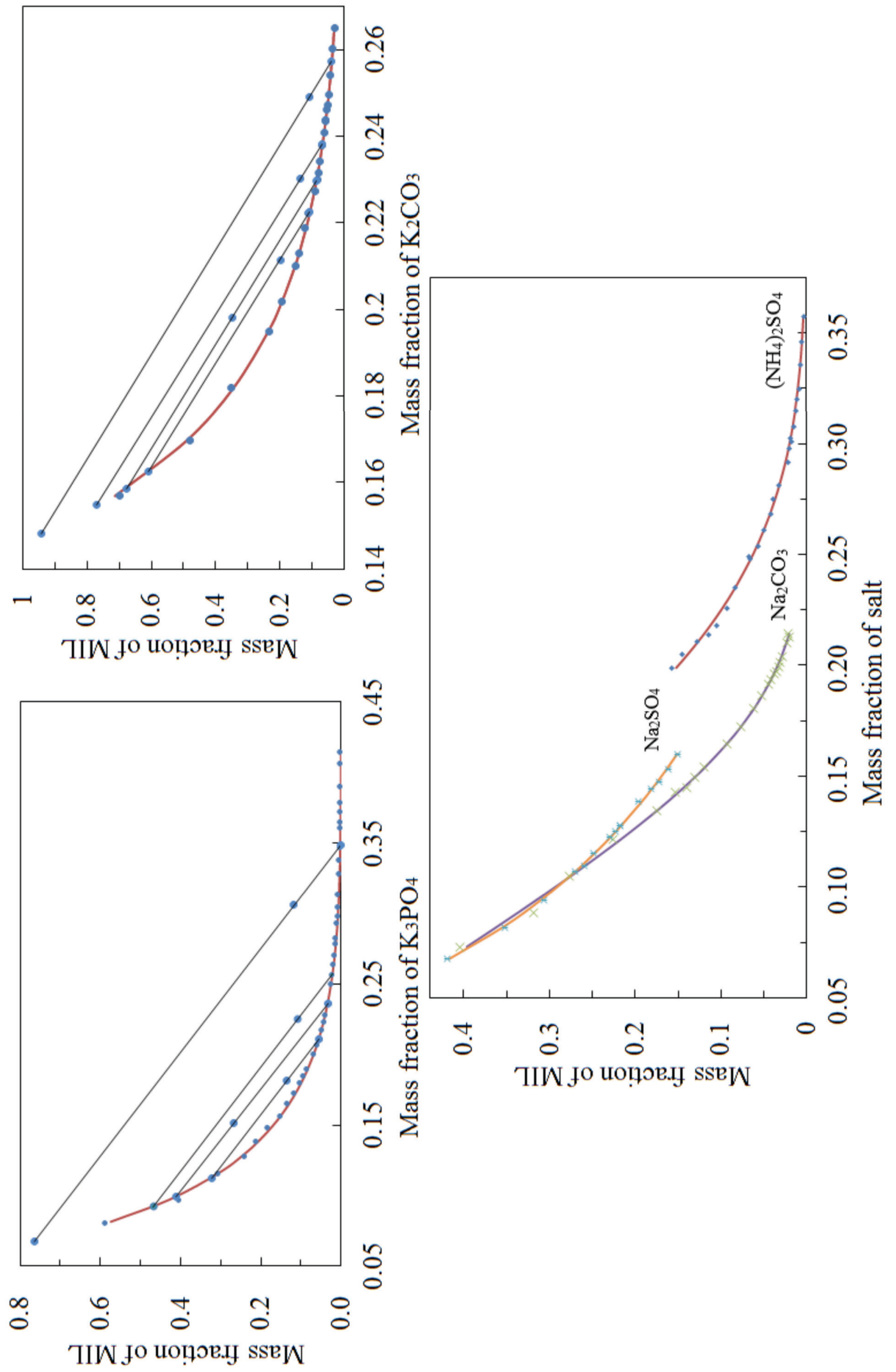


Fig.7

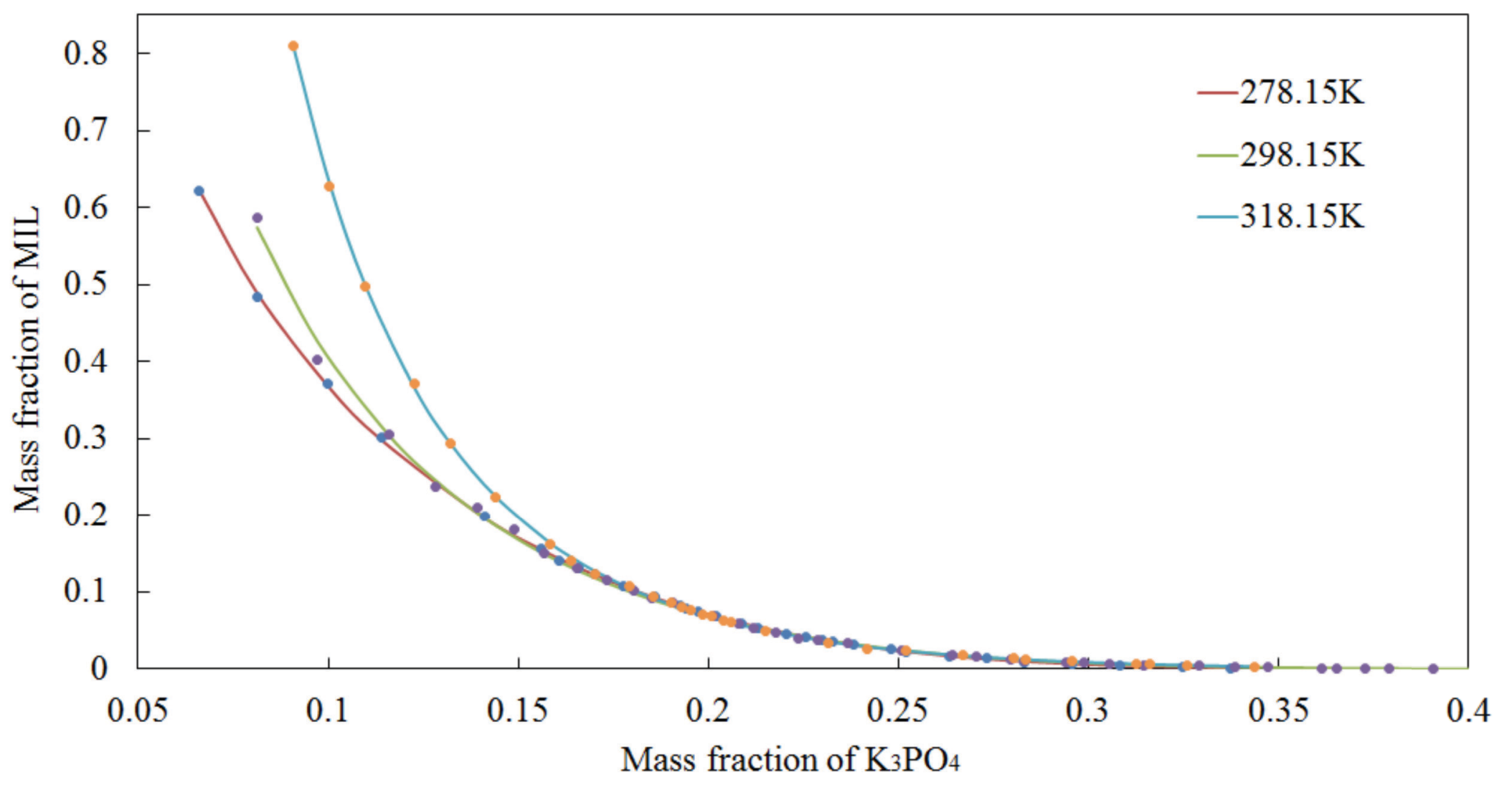


Fig. 8
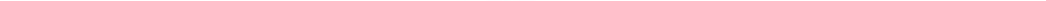
Fig.9
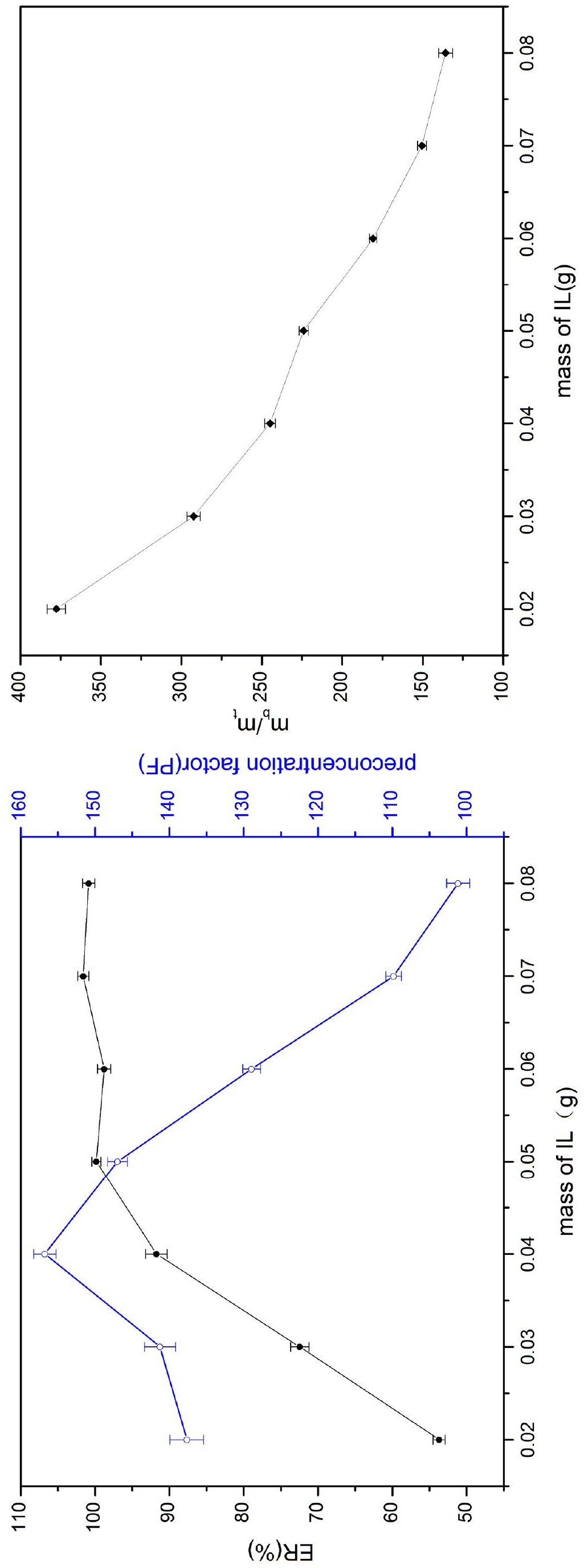
Fig.10
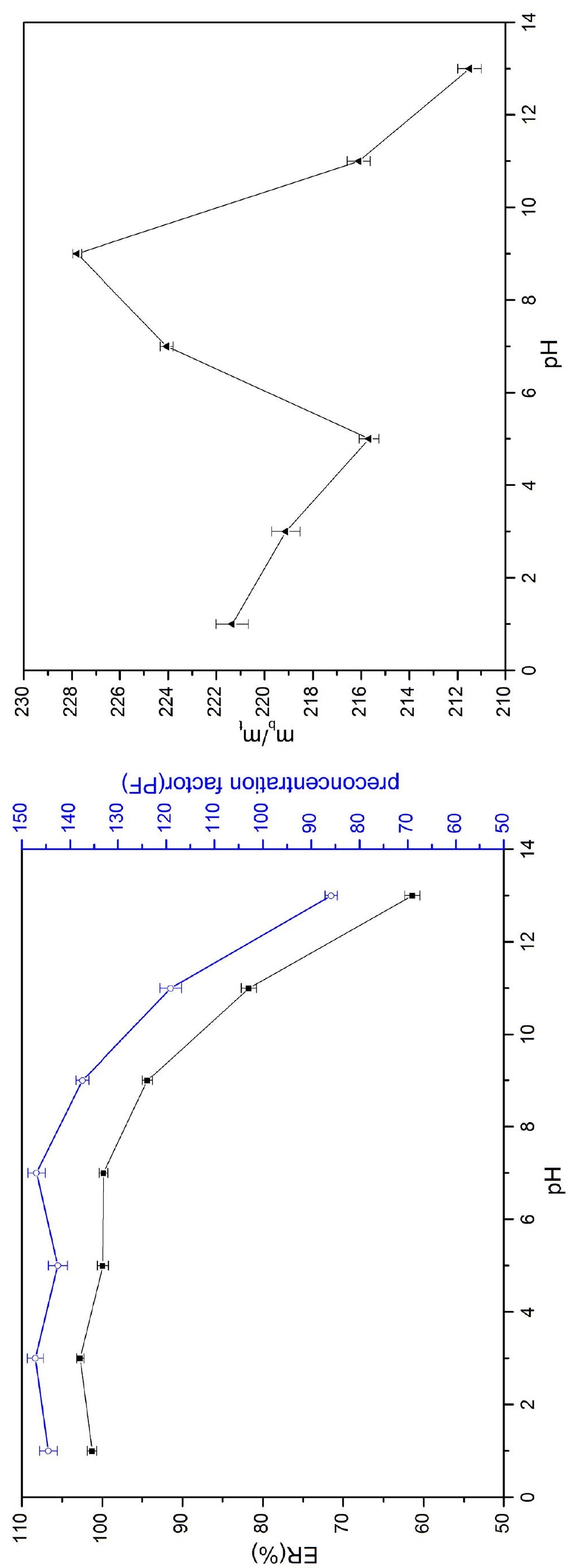


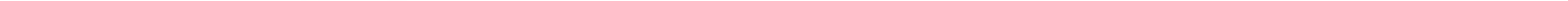

